\title{
PRUEBA DE APTITUD TÉCNICA EN LA MAGNITUD DE TEMPERATURA PARA LABORATORIOS DE CALIBRACIÓN
}

\author{
Ignacio López Hernández
}

\section{Resumen}

Se presentan los resultados obtenidos en el ensayo de aptitud LACOMET-IC-05-2009, realizado por el Laboratorio Costarricense de Metrología (LACOMET), mediante la calibración y medición - por parte de los laboratorios participantes - de un termómetro previamente calibrado por LACOMET. El alcance de medición fue $0{ }^{\circ} \mathrm{C}$ a $100{ }^{\circ} \mathrm{C}$.

En este ejercicio de ensayo de aptitud participaron ocho laboratorios secundarios de calibración. El instrumento itinerante utilizado fue un termómetro de líquido de vidrio marca KESSLER, modelo ASTM 9C, Serie 504079, con una división de escala de $0,5^{\circ} \mathrm{C}$ y de inmersión parcial de $57 \mathrm{~mm}$.

LACOMET se encargó de la coordinación y del análisis de la información proporcionada por los laboratorios participantes. En este ensayo de aptitud participaron laboratorios secundarios que necesitan cumplir con requisitos exigidos por la norma INTE-ISO/IEC 17025:2005 (INTECO, 2005), relativos a materia de comparaciones. También se presenta información adicional que puede significar oportunidades de mejora para los laboratorios participantes.

Con este tipo de ejercicios y de divulgación de resultados se cumplen los objetivos que tiene el Laboratorio Costarricense de Metrología (LACOMET) de verificar y armonizar la competencia técnica de los laboratorios nacionales de calibración, con el fin de promover una red de laboratorios con mediciones confiables y con un nivel de concordancia entre ellos, dentro de la mejor capacidad de medición declarada por cada laboratorio.

Palabras claves: metrología, calibración, temperatura, ensayo de aptitud, competencia técnica.

\begin{abstract}
This document shows the results of aptitude test LACOMET-IC-05-2009, made by the Laboratorio Costarricense de Metrología (LACOMET), which was realized by the calibration and measurement - by the participant laboratories - of a thermometer previously calibrated by LACOMET. The measurement reach went $0{ }^{\circ} \mathrm{C}$ to $100{ }^{\circ} \mathrm{C}$.

In this exercise of aptitude test, eight secondary laboratories of calibration participated. The traveling instrument used was a liquid in glass thermometer of the KESSLER brand, model ASTM 9C, Series 504079, with division of scale of $0.5^{\circ} \mathrm{C}$ and a partial immersion of $57 \mathrm{~mm}$.

LACOMET was in charge of the coordination and the analysis of the information provided by the participant laboratories. In this test of aptitude participated secondary laboratories that need to complete requirements demanded by norm INTE-ISO/IEC 17025:2005 (INTECO, 2005), regarding matter of comparisons. Also, additional information that appears they can mean opportunities of improvement for the participant laboratories.

With this type of exercises and spreading of results, the objectives of the Laboratorio Costarricense de Metrología (LACOMET) of verify and to harmonize the technical competition of the national laboratories of calibration are fulfilled, with the purpose of promote a network of laboratories with reliable measurements and a level of agreement among them, within the best capacity of measurement declared by each laboratory.
\end{abstract}

Keywords: metrology, calibration, temperature, measurement, comparison, aptitude test.

Recibido: 29 de enero del 2010 -Aprobado: 11 de febrero del 2010.

\section{INTRODUCCIÓN}

Este documento resume los resultados del ensayo de aptitud realizado por el Laboratorio Costarricense de Metrología como laboratorio piloto y los laboratorios nacionales que dan servicio de calibración, los cuales realizaron esfuerzos significativos para cumplir con el requisito técnico de las comparaciones. El objetivo de la práctica en cuestión fue determinar la compatibilidad y 
el grado de concordancia entre las mediciones de la red de laboratorios secundarios (acreditados y no acreditados). El país requiere de un diagnóstico del nivel metrológico en la magnitud de temperatura para homologar criterios técnicos.

El programa de este ensayo de aptitud fue desarrollado y propuesto por el Laboratorio Costarricense de Metrología en coordinación con los laboratorios participantes. Las mediciones se iniciaron el 14 de setiembre del 2009 y finalizaron el 17 de diciembre del 2010.

Durante el desarrollo del ensayo de aptitud, el Laboratorio de Temperatura de LACOMET realizó una medición inicial del termómetro viajero. Cada uno de los laboratorios participantes que se muestran en el Cuadro 1, realizaron las mediciones en sus sistemas de medición siguiendo para tal efecto los lineamientos dados en el protocolo de medición Prueba de Aptitud Técnica para Laboratorios de Calibración LACOMET -IC-05-2009.

\section{DESARROLLO}

El ensayo de aptitud fue realizado siguiendo para tal efecto los lineamientos del protocolo Prueba de Aptitud Técnica para Laboratorios de Calibración LACOMET-IC05-2009. Los resultados de cada laboratorio se enviaron a LACOMET para su revisión y el correspondiente análisis para su evaluación.

LACOMET fue el encargado de generar los valores de referencia para realizar la evaluación del termómetro itinerante y del ensayo de aptitud individual de cada laboratorio. En este documento, la evaluación se resume en una gráfica de error normalizado, elaborada con la información generada de cada laboratorio.

\subsection{Laboratorios participantes}

En el Cuadro 1 se muestra la lista de laboratorios participantes en este ensayo de aptitud y el programa de mediciones propuesto aparece en la Figura 1.

\subsection{Termómetro utilizado}

Para efectos del desarrollo de la comparación, ha sido acordada la utilización de un termómetro de líquido de vidrio con las siguientes características:

$\begin{array}{ll}\text { - } & \text { Marca KESSLER } \\ \text { - } & \text { Modelo ASTM 9C } \\ \text { - } & \text { Serie 504079 } \\ \text { - } & \text { División de escala de } 0,5^{\circ} \mathrm{C} \\ \text { - } & \text { Inmersión parcial de } 57 \mathrm{~mm}\end{array}$

Cuadro 1. Lista de laboratorios participantes.

Laboratorio Temperatura - LACOMET (Laboratorio referencia)

Met-Cal-Engineering Services

Laboratorio Metrológico Empresarial RECOPE

A1 Calibration Laboratory S. A.

Metrología Consultores S. A.

Laboratorio de Calibración de Bridgestone CR

Calibraciones de América (CAMÉRICA S. A.)

LABCAL-UCR

Desarrollos Latinoamericanos de Metrología Integrada, S. A. (DMI Metrología)

Fuente: (El autor, LACOMET. Laboratorio de Temperatura) 


\subsection{Protocolo de medición}

Cada laboratorio participante envió a LACOMET los datos de las mediciones así como el informe de calibración, tomando en cuenta la siguiente información:

i) los datos de las mediciones tal y como fueron obtenidas del proceso de medición;

ii) el error relativo del termómetro itinerante respecto a la medición realizada por el laboratorio;

iii) la incertidumbre declarada o acreditada del sistema de medición de cada laboratorio;

iv) la incertidumbre expandida de la calibración y, v) las fuentes de incertidumbre adicionales consideradas de acuerdo con el procedimiento de cada laboratorio.

Se usaron las recomendaciones establecidas por (BIPM, IEC, IFCC, ISO, IUPAC, IUPAP \& OIML, 1995) para el análisis de los resultados.

\section{RESULTADOS}

Los resultados de la comparación se muestran en los Cuadros 2, 3 y 4 y en las Figuras 2, 3 y 4. En los Cuadros se presentan el error no normalizado, la incertidumbre expandida y el error normalizado de cada laboratorio. En las figuras se presentan solo los errores no

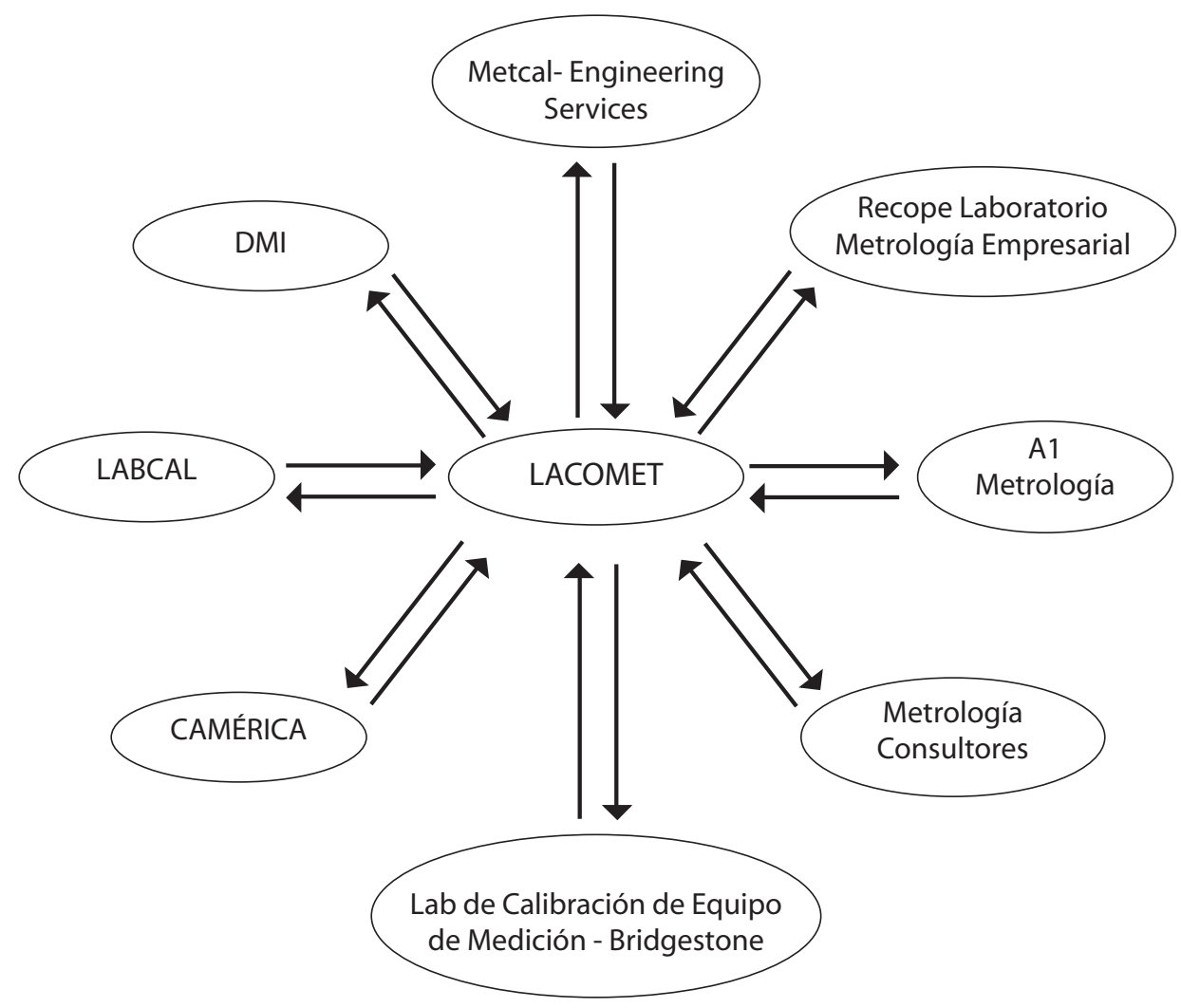

Figura 1. Ruta del termómetro itinerante. Fuente: (Rodríguez, LACOMET). 
normalizados con su banda de incertidumbre. El grado de equivalencia entre los resultados de las mediciones de los laboratorios fue determinado a partir del error normalizado de acuerdo con la ecuación (1).

$$
E_{n}=\frac{\left|E_{L}-E_{L A}\right|}{\sqrt{U_{L}^{2}+U_{L A}^{2}}}
$$

\section{Donde:}

$E_{L} \quad$ es el valor reportado por el laboratorio participante.

$E_{L A} \quad$ es el valor reportado por el LACOMET.

$U_{L} \quad$ es la incertidumbre expandida, al $95 \%$ de confianza, asociada al cálculo del error reportado por el laboratorio participante.

$U_{L A} \quad$ es la incertidumbre expandida, al $95 \%$ de confianza, asociada al cálculo del error reportado por el LACOMET.

Los criterios de análisis son:

$\begin{array}{lll}\text { En } & \leq 1 & \text { Resultado: Satisfactorio } \\ \text { En } & >1 & \text { Resultado: Cuestionable }\end{array}$

Según los resultados que se detallan en el Cuadro 2 , que corresponde a mediciones en cero grados Celsius, se observa que los laboratorios 1,3, 5, 7,8 y 9 presentan errores normalizados menores a 1 , esto implica que son satisfactorios. Los laboratorios 4 y 10 tienen errores normalizados superiores a 1 , esto significa que sus resultados no son satisfactorios.

De acuerdo con los resultados que se detallan en el Cuadro 3, que corresponde a mediciones en cincuenta grados Celsius, se observa que los laboratorios 3, 4, 5, 7, 8, 9 y 10 presentan errores normalizados menores a 1 , esto significa que son equivalentes a los resultados del laboratorio de referencia. El laboratorio 1 tiene errores normalizados superiores a 1 , esto significa que su resultado no es satisfactorio en las mediciones realizadas a esta temperatura.

En los resultados que se detallan en el Cuadro 4 , que corresponde a mediciones en cien grados Celsius, se observa que los laboratorios $1,3,5,7$, 8 y 9 presentan errores normalizados menores a 1 , lo cual deja en evidencia que sus métodos son adecuados. Los laboratorios 4 y 10 tienen errores normalizados superiores a 1 , esto significa que sus resultados no son satisfactorios.

Las Figuras 2, 3 y 4 confirman que laboratorios con errores normalizados superiores a 1 aparecen fuera del intervalo (error $\pm \mathrm{U}$ ) ${ }^{\circ} \mathrm{C}$ reportado por LACOMET, que es el laboratorio de referencia.

Cuadro 2. Valor de los errores normalizados en $0{ }^{\circ} \mathrm{C}$.

\begin{tabular}{cccc}
\hline Laboratorio & Error del laboratorio & Incertidumbre & $\begin{array}{c}\text { Error } \\
\text { normalizado }\end{array}$ \\
\hline & $\left({ }^{\circ} \mathrm{C}\right)$ & $\left({ }^{\circ} \mathrm{C}\right)$ & \\
\hline 01 & $-0,25$ & 0,15 & 0,02 \\
03 & $-0,25$ & 0,16 & 0,02 \\
04 & 0,03 & 0,14 & 1,38 \\
05 & $-0,23$ & 0,15 & 0,08 \\
LACOMET & $-0,25$ & 0,14 & REFERENCIA \\
07 & $-0,20$ & 0,30 & 0,14 \\
08 & $-0,10$ & 0,30 & 0,44 \\
09 & $-0,04$ & 0,25 & 0,72 \\
10 & 0,00 & 0,14 & 1,23 \\
\hline
\end{tabular}




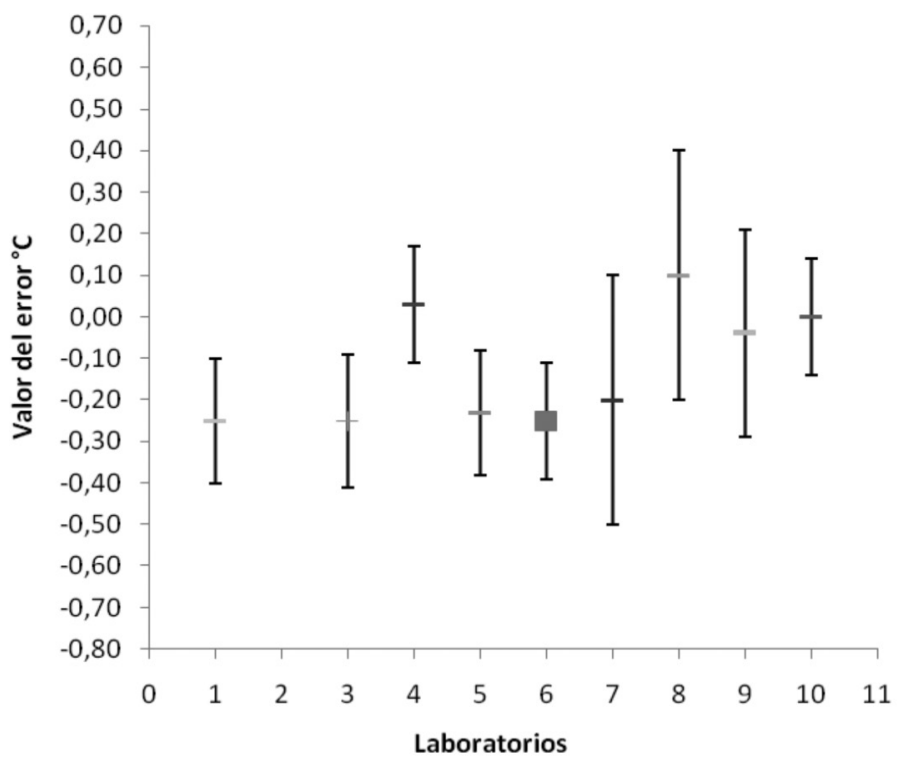

Figura 2. Valor de los errores en $0{ }^{\circ} \mathrm{C}$.

Nota: LACOMET corresponde al laboratorio 6 Fuente: (El autor, LACOMET. Laboratorio de Temperatura).

Cuadro 3. Temperatura nominal $50{ }^{\circ} \mathrm{C}$.

\begin{tabular}{cccc}
\hline Laboratorio & $\begin{array}{c}\text { Error del } \\
\text { laboratorio }\end{array}$ & Incertidumbre & Error normalizado \\
\hline 01 & $\left({ }^{\circ} \mathrm{C}\right)$ & $\left({ }^{\circ} \mathrm{C}\right)$ & \\
\hline 03 & $-0,28$ & 0,15 & 1,31 \\
04 & 0,18 & 0,16 & 0,87 \\
05 & 0,01 & 0,14 & 0,08 \\
LACOMET & 0,04 & 0,20 & 0,19 \\
07 & $-0,01$ & 0,15 & REFERENCIA \\
08 & $-0,29$ & 0,30 & 0,85 \\
09 & $-0,10$ & 0,30 & 0,28 \\
10 & $-0,26$ & 0,25 & 0,87 \\
& 0,00 & 0,14 & 0,04 \\
\hline
\end{tabular}

Fuente: (El autor, Lacomet. Laboratorio de Temperatura). 


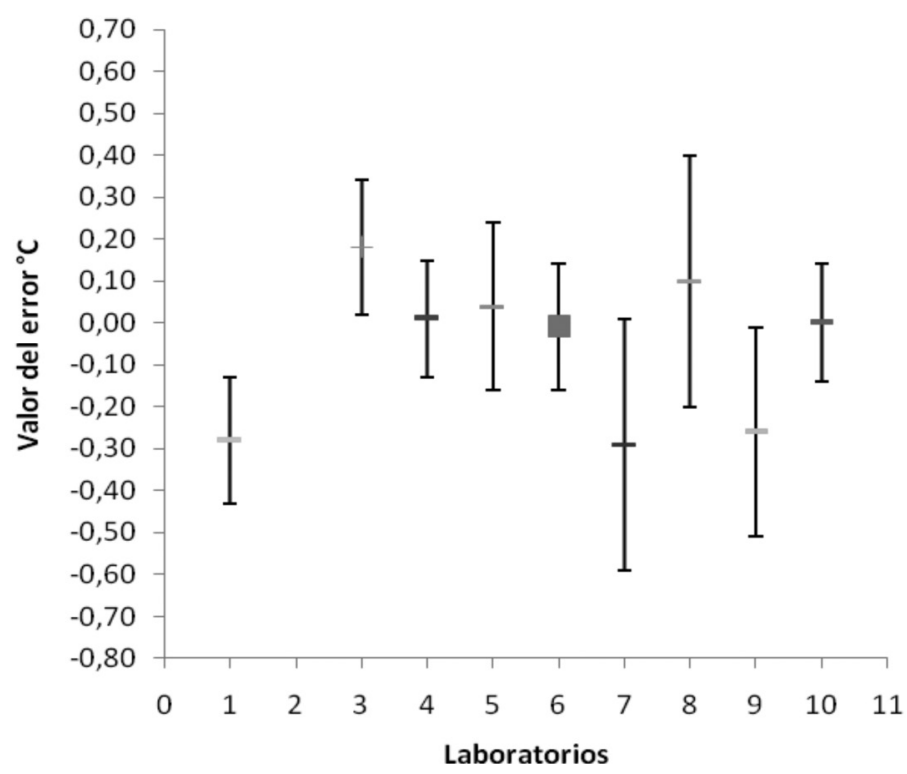

Figura 3. Valor de los errores en $50^{\circ} \mathrm{C}$.

Nota: LACOMET corresponde al laboratorio 6

Fuente: (El autor, LACOMET. Laboratorio de Temperatura).

Cuadro 4. Valor de los errores normalizados en $100{ }^{\circ} \mathrm{C}$.

\begin{tabular}{cccc}
\hline Laboratorio & Error del laboratorio & Incertidumbre & Error normalizado \\
\hline & $\left({ }^{\circ} \mathrm{C}\right)$ & $\left({ }^{\circ} \mathrm{C}\right)$ & \\
01 & 0,37 & 0,15 & 0,51 \\
03 & 0,25 & 0,16 & 0,04 \\
04 & 0,01 & 0,14 & 1,26 \\
05 & 0,32 & 0,40 & 0,13 \\
LACOMET & 0,26 & 0,15 & REFERENCIA \\
07 & 0,00 & 0,30 & 0,79 \\
08 & 0,10 & 0,30 & 0,49 \\
09 & $-0,02$ & 0,25 & 0,97 \\
10 & $-0,01$ & 0,14 & 1,35 \\
\hline
\end{tabular}

Fuente: (El autor, LACOMET. Laboratorio de Temperatura). 


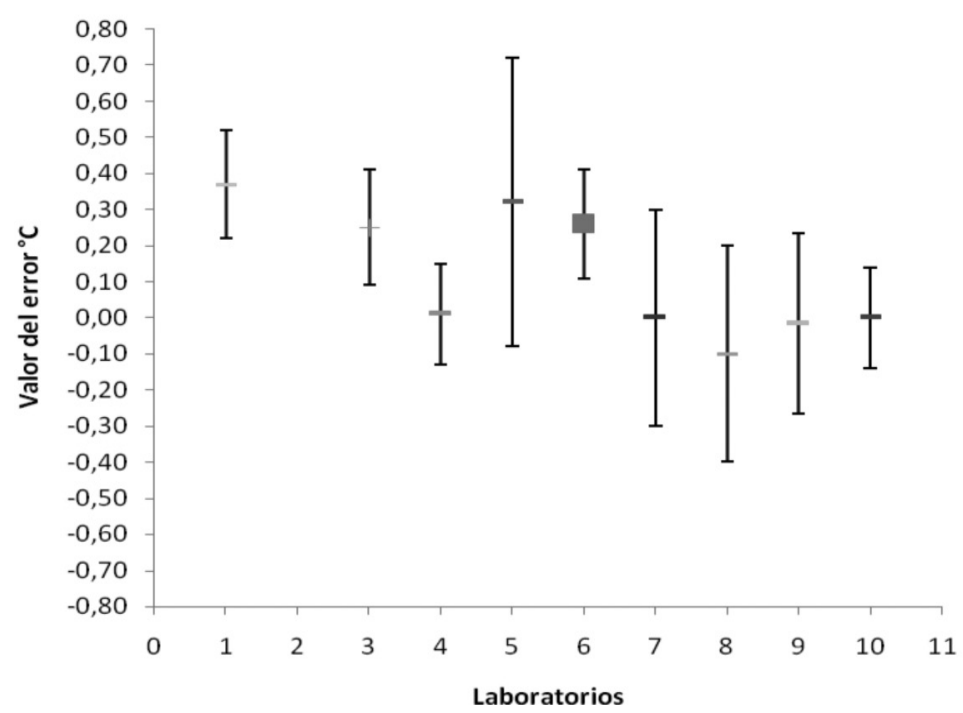

Figura 4. Valor de los errores en $100{ }^{\circ} \mathrm{C}$.

Nota: LACOMET corresponde al laboratorio 6

Fuente: (el autor, LACOMET. Laboratorio de Temperatura).

\section{CONCLUSIONES}

1. Este ejercicio comparativo es el inicio de un proceso que busca extenderse a más instrumentos de medición de temperatura con mejores exactitudes.

2. Los laboratorios que mostraron equivalencia con los resultados del laboratorio de referencia (LACOMET) en todas las temperaturas que fueron medidas son los laboratorios 3, 5, 7, 8 y 9. Estos laboratorios tienen la capacidad de generar resultados técnicamente válidos.

3. El laboratorio 1 generó un error normalizado mayor a 1 en sus mediciones en la temperatura de cincuenta grados Celsius; se recomienda revisar el cálculo de la corrección por columna emergente que generó la no equivalencia en sus mediciones realizadas a esa temperatura.

4. El laboratorio 4 y el 10 presentan errores normalizados mayores a 1 en sus mediciones de las temperaturas de $0{ }^{\circ} \mathrm{C}$ y $100{ }^{\circ} \mathrm{C}$, se recomienda revisar el cálculo de la corrección por columna emergente que generó la no equivalencia en las mediciones realizadas a esas temperaturas.

5. Con la culminación de esta prueba de aptitud técnica se cumplieron los objetivos planteados. Se ha iniciado un proceso de retroalimentación necesario para homologar criterios técnicos y se han dado los primeros pasos para conformar un comité técnico nacional en la magnitud de temperatura. Este ejercicio comparativo revela la capacidad técnica de los participantes necesaria para fortalecer sus capacidades de medición y calibración, da confianza de la calidad de sus resultados a los clientes y asegura la cadena de trazabilidad. Estos resultados también son valiosos para la labor de los evaluadores del Ente Costarricense de Acreditación (ECA) 
que evalúa los procesos de acreditación, labor necesaria para garantizar el funcionamiento del Sistema Nacional de Calidad.

6. Esta comparación puso en evidencia la necesidad de desarrollar en el país la metrología de investigación para buscar técnicas e instrumentos de medición de temperatura más prácticos y eficientes. El aporte de las universidades, el Estado y la empresa privada es indispensable para iniciar este proceso.

\section{AGRADECIMIENTOS}

El Laboratorio Costarricense de Metrología agradece a todos los laboratorios secundarios que participaron en esta actividad, la cual contribuye a fortalecer la infraestructura metrológica nacional.

\section{REFERENCIAS BIBLIOGRÁFICAS}

ASTM (American Society for Testing Materials). ASTM E1-07 Standard specification for ASTM liquid-in-glass thermometers.

BIPM, IEC, IFCC, ISO, IUPAC, IUPAP \& OIML. (1995). Guide to the expression of uncertainty in measurement. First edition. International Organization for Standarization: Switzerland.

CENAM (Centro Nacional de Metrología). GUM "Guide to the expression of uncertainty in measurement ISO-IEC-OIML-BIPM" para el análisis de los resultados.
CENAM (Centro Nacional de Metrología). Interlaboratory mass comparison between laboratories belonging to SIM Sub-Region coordinate by CENAM, Mexico. Final Report SIM.7.31a-SIM.7.31b, CENAM2006, Sec 8, p. 3.

ECA (Ente Costarricense de Acreditación). (2006). ECA-MC-P17 Política y criterios para la participación en ensayos/pruebas de aptitud y otras comparaciones para los laboratorios. Versión 1. Ente Costarricense de Acreditación, San José: Editor.

INTECO (Instituto Costarricense de Normas Técnicas). (2005). INTE-ISO/IEC 17025:2005. Requisitos generales para la competencia de laboratorios de ensayo y calibración. (1a ed.) San José: Editor.

INTECO (Instituto Costarricense de Normas Técnicas). (2000). INTE ISO/IEC Guía 43-1:2000. Ensayo de aptitud por comparaciones interlaboratorios. Parte 1: Desarrollo y funcionamiento de programas de ensayos de aptitud. San José: Editor.

\section{SOBRE EL AUTOR}

\section{Ignacio López Hernández}

Licenciado en Ingeniería Industrial

Laboratorio Costarricense de Metrología

Metrología Física

Teléfonos: (506) 2283-6580

Facsímil: (506) 2283-5133

Correo electrónico: ilopez@lacomet.go.cr 